PROCEEDINGS OF THE

AMERICAN MATHEMATICAL SOCIETY

Volume 136, Number 10, October 2008, Pages 3621-3625

S 0002-9939(08)09358-1

Article electronically published on May 19, 2008

\title{
THE FATOU SET FOR CRITICALLY FINITE MAPS
}

\author{
FENG RONG
}

(Communicated by Mei-Chi Shaw)

\begin{abstract}
It is a classical result in complex dynamics of one variable that the Fatou set for a critically finite map on $\mathbf{P}^{1}$ consists of only basins of attraction for superattracting periodic points. In this paper, we deal with critically finite maps on $\mathbf{P}^{k}$. We show that the Fatou set for a critically finite map on $\mathbf{P}^{2}$ consists of only basins of attraction for superattracting periodic points. We also show that the Fatou set for a $k$-critically finite map on $\mathbf{P}^{k}$ is empty.
\end{abstract}

\section{INTRODUCTION}

A holomorphic map $f: \mathbf{P}^{k} \rightarrow \mathbf{P}^{k}$ is said to be critically finite if every component of the critical set for $f$ is periodic or preperiodic. In [5], Thurston has given a topological classification of critically finite maps on $\mathbf{P}^{1}$. And it is well known that the Fatou set for a critically finite map on $\mathbf{P}^{1}$ consists of only basins of attraction for superattracting periodic points, i.e. points $p$ with $f^{n}(p)=p$ and $\left(f^{n}\right)^{\prime}(p)=0$ for some $n \in \mathbf{N}$ (see 4 ). In this paper, we show that the same is also true for critically finite maps on $\mathbf{P}^{2}$. More precisely, we have the following

Theorem 1.1. If $f: \mathbf{P}^{2} \rightarrow \mathbf{P}^{2}$ is a critically finite holomorphic map, then the Fatou set for $f$ consists of only basins of attraction for superattracting periodic points.

With some extra assumptions, the above result has been obtained by Fornæss and Sibony (2]).

We will also study critically finite maps on $\mathbf{P}^{k}$. In particular, we obtain the following theorem (see Section 2 for precise definitions).

Theorem 1.2. Let $f: \mathbf{P}^{k} \rightarrow \mathbf{P}^{k}$ be a holomorphic map. If $f$ is $k$-critically finite, then the Fatou set for $f$ is empty.

For 1 -critically finite maps on $\mathbf{P}^{1}$ and 2 -critically finite maps on $\mathbf{P}^{2}$, this was proved by Thurston ([5]) and Ueda ([6]), respectively.

The author would like to thank John Erik Fornæss for his advice and encouragement.

\section{The FAtou SET FOR CRITICALly Finite MAPS}

Let $f: \mathbf{P}^{k} \rightarrow \mathbf{P}^{k}$ be a holomorphic map of (algebraic) degree $d>1$.

Received by the editors July 17, 2007, and, in revised form, September 13, 2007.

2000 Mathematics Subject Classification. Primary 32H50.

(C)2008 American Mathematical Society 
Let $C_{1}$ be the critical set of $f$ given by

$$
C_{1}=\left\{p \in \mathbf{P}^{k} \mid \operatorname{rank}(d f(p))<k\right\},
$$

where $d f(p)$ denotes the differential of $f$ at $p$.

We define the post-critical set $D_{1}$ of $f$ by

$$
D_{1}=\bigcup_{j=1}^{\infty} f^{j}\left(C_{1}\right),
$$

and the $\omega$-limit set $E_{1}$ of $f$ by

$$
E_{1}=\bigcap_{j=1}^{\infty} f^{j}\left(\overline{D_{1}}\right) .
$$

By definition, a holomorphic map $f$ on $\mathbf{P}^{k}$ is critically finite if the post-critical set $D_{1}$ is an analytic (hence algebraic) set in $\mathbf{P}^{k}$. This is equivalent to saying that there is an integer $l \geq 1$ such that $D_{1}=\bigcup_{j=1}^{l} f^{j}\left(C_{1}\right)$. Hence, in the critically finite case, the set $D_{1}$ is an algebraic set of pure codimension 1 .

Let us take a closer look at the structure of the post-critical set $D_{1}$ and the $\omega$-limit set $E_{1}$. If $f$ is critically finite, then $f^{j-1}\left(D_{1}\right)=\bigcup_{l=j}^{\infty} f^{l}\left(C_{1}\right), j=1,2, \cdots$, is a descending sequence of algebraic sets. Hence there is an integer $l_{1} \geq 1$ such that $f^{l_{1}-1}\left(D_{1}\right)=f^{l_{1}}\left(D_{1}\right)=\cdots$. Consequently $E_{1}=f^{l_{1}-1}\left(D_{1}\right)$ is an algebraic set of pure codimension 1 . We can decompose $E_{1}$ into $E_{1}^{\prime} \cup F_{1}$, where $F_{1}$ consists of those components in a critical cycle. (A periodic component $L$ is said to be in a critical cycle if at least one of the forward images of $L$ under $f$ is contained in the critical set for $f$.)

Definition 2.1. Let $f: \mathbf{P}^{k} \rightarrow \mathbf{P}^{k}$ be a holomorphic map. The map $f$ is said to be critically finite of order 1 if $D_{1}$, hence $E_{1}$, is algebraic. And $f$ is said to be 1-critically finite if $C_{1}$ and $E_{1}$ have no common irreducible component, i.e. $F_{1}=\emptyset$.

We can now make the following inductive definition (cf. [3]).

Definition 2.2. Let $f: \mathbf{P}^{k} \rightarrow \mathbf{P}^{k}$ be a holomorphic map. Suppose $f$ is critically finite of order $n-1,1<n \leq k$. Denote $C_{n}=C_{1} \cap E_{n-1}, D_{n}=\bigcup_{j=1}^{\infty} f^{j}\left(C_{n}\right)$, and $E_{n}=\bigcap_{j=1}^{\infty} f^{j}\left(\overline{D_{n}}\right)$. We say that $f$ is critically finite of order $n$ if $D_{n}$, hence $E_{n}$, is algebraic. Let $l_{n}$ be the least integer such that $E_{n}=f^{l_{n}-1}\left(D_{n}\right)=\bigcup_{j=l_{n}}^{\infty} f^{j}\left(C_{n}\right)$. We can decompose $E_{n}$ into $E_{n}^{\prime} \cup F_{n}$, where $F_{n}$ consists of those components, of codimension less or equal to $n$, in a critical cycle. If in addition $f$ is $(n-1)-$ critically finite, then we say that $f$ is $n$-critically finite if $E_{n}$ has no irreducible component contained in $C_{1}$, i.e. $F_{n}=\emptyset$.

Remark 2.3. A critically finite map is by definition critically finite of order 1 . Jonsson (3, Remark 2.10]) noted that a critically finite map on $\mathbf{P}^{2}$ is always strictly critically finite in the sense of Fornæss and Sibony (2]). Recall that a critically finite map $f$ is said to be strictly critically finite if $f$, when restricted to each irreducible periodic component of $E_{1}$, is also critically finite. We will use this remark implicitly in the proof of Theorem 1.1.

Before we go further, let us recall some definitions and results from [6].

Definition 2.4. Let $f: \mathbf{P}^{k} \rightarrow \mathbf{P}^{k}$ be a holomorphic map and let $U$ be a Fatou component, i.e. a connected component of the Fatou set for $f$. A holomorphic 
map $\varphi: U \rightarrow \mathbf{P}^{k}$ is called a limit map on $U$ if there is a sequence $\left\{f^{n_{j}} \mid U\right\}$ which converges to $\varphi$ uniformly on compact sets in $U$. A point $q \in \mathbf{P}^{k}$ is called a Fatou limit point if there is a limit map $\varphi$ on a Fatou component $U$ such that $q \in \varphi(U)$. The set of all Fatou limit points is called the Fatou limit set.

Definition 2.5. A Fatou component $U$ is called a rotation domain if the identity map $i d_{U}: U \rightarrow U$ is a limit map on $U$.

Definition 2.6. A point $q \in \mathbf{P}^{k}$ is said to be a point of bounded ramification with respect to $f$ if the following conditions are satisfied:

(i) There is a neighborhood $W$ of $q$ such that $D_{1} \cap W$ is an analytic subset of $W$.

(ii) There exists an integer $l$ such that, for every integer $j>0$ and every $p \in$ $f^{-j}(q)$, the cardinality $\sharp(I)$ of the set

$$
I=\left\{i \mid 0 \leq i \leq j-1, f^{i}(p) \in C_{1}\right\}
$$

is not greater than $l$.

The following two theorems by Ueda are crucial.

Theorem 2.7 ([6, Theorem 4.8]). Suppose that $q \in \mathbf{P}^{k}$ is a point of bounded ramification and also a Fatou limit point. Then $q$ is contained in a rotation domain.

Theorem 2.8 ([6, Proposition 5.1, (1)]). If $f: \mathbf{P}^{k} \rightarrow \mathbf{P}^{k}$ is critically finite, then there is no rotation domain.

Remark 2.9. Since the set $D_{1}$ is an analytic set in the critically finite case, condition (i) in Definition 2.6 is automatically true. So we only need to check condition (ii) in Definition 2.6 to see if a point $p \in \mathbf{P}^{k}$ is of bounded ramification.

We need the following lemma, whose proof is an elaboration of the proof of 6 , Lemma 5.7].

Lemma 2.10. Let $f: \mathbf{P}^{k} \rightarrow \mathbf{P}^{k}$ be a holomorphic map. If $f$ is critically finite of order $n, 1 \leq n<k$, then every point in $\mathbf{P}^{k} \backslash E_{n}$ is a point of bounded ramification. If $f$ is critically finite of order $k$, then every point in $\mathbf{P}^{k} \backslash F_{k}$ is a point of bounded ramification.

Proof. First assume that $f$ is critically finite of order $n, 1 \leq n<k$. Let $q \in \mathbf{P}^{k} \backslash E_{n}$ and let $p \in f^{-j}(q)$ for some integer $j>0$. By Remark 2.9, we only need to show that the cardinality $\sharp(I)$ of the set

$$
I=\left\{i \mid 0 \leq i \leq j-1, f^{i}(p) \in C_{1}\right\}
$$

is not greater than some integer $l>0$. Let

$$
\begin{gathered}
I_{m}=\left\{i \mid 0 \leq i \leq j-1, f^{i}(p) \in C_{m} \backslash C_{m+1}\right\}, \quad m=1, \cdots, n-1, \\
I_{n}=\left\{i \mid 0 \leq i \leq j-1, f^{i}(p) \in C_{n}\right\} .
\end{gathered}
$$

We claim that $\sharp\left(I_{m}\right) \leq l_{m}, m=1, \cdots, n$.

For each $1 \leq m<n$, suppose that $I_{m}$ is non-empty and let $i_{m}$ be the least index in $I_{m}$. Then $f^{i_{m}}(p) \in C_{m}$. For $i \geq i_{m}+l_{m}$, we have $f^{i}(p) \in E_{m}$ and hence $f^{i}(p) \notin C_{m} \backslash C_{m+1}$. Thus $I_{m}$ is a subset of $\left\{i_{m}, \cdots, i_{m}+l_{m}-1\right\}$, and $\sharp\left(I_{m}\right) \leq l_{m}$.

Now suppose that $I_{n}$ is non-empty and let $i_{n}$ be the least index in $I_{n}$. Then $f^{i_{n}}(p) \in C_{n}$. For $i \geq i_{n}+l_{n}$, we have $f^{i}(p) \in E_{n}$. Since $f^{j}(p)=q \notin E_{n}$, we have $i_{n}+l_{n}>j$. Thus $I_{n}$ is a subset of $\left\{i_{n}, \cdots, i_{n}+l_{n}-1\right\}$ and $\sharp\left(I_{n}\right) \leq l_{n}$. 
Next assume that $f$ is critically finite of order $k$. Let $q \in \mathbf{P}^{k} \backslash F_{k}$ and let $p \in$ $f^{-j}(q)$ for some integer $j>0$. Let

$$
\begin{gathered}
I_{m}=\left\{i \mid 0 \leq i \leq j-1, f^{i}(p) \in C_{m} \backslash C_{m+1}\right\}, \quad m=1, \cdots, k-1, \\
I_{k}=\left\{i \mid 0 \leq i \leq j-1, f^{i}(p) \in C_{k}\right\} .
\end{gathered}
$$

For the same reason as above we have that $\sharp\left(I_{m}\right) \leq l_{m}$ for $1 \leq m<k$. Now suppose that $I_{k}$ is non-empty and let $i_{k}$ be the least index in $I_{k}$. Then $f^{i_{k}}(p) \in C_{k}$. For $i \geq i_{k}+l_{k}$, we have $f^{i}(p) \in E_{k}$. Note that $f\left(F_{k}\right)=F_{k}$ and $\left(E_{k} \backslash F_{k}\right) \cap C_{k}=\emptyset$. Since $f^{j}(p)=q \notin F_{k}$, we have $i_{k}+l_{k}>j$. Thus $I_{k}$ is a subset of $\left\{i_{k}, \cdots, i_{k}+l_{k}-1\right\}$ and $\sharp\left(I_{k}\right) \leq l_{k}$.

Combining this lemma with Theorems 2.7 and 2.8, we obtain the following result, which generalizes [6, Theorem 5.8].

Theorem 2.11. Let $f: \mathbf{P}^{k} \rightarrow \mathbf{P}^{k}$ be a holomorphic map. If $f$ is critically finite of order $n, 1 \leq n<k$, then the Fatou limit set is contained in $E_{n}$. If $f$ is critically finite of order $k$, then the Fatou limit set is contained in $F_{k}$.

By definition, a $k$-critically finite map on $\mathbf{P}^{k}$ has $F_{k}=\emptyset$. Therefore we obtain Theorem 1.2 as a corollary to the above theorem.

Now we turn to critically finite maps on $\mathbf{P}^{2}$. We say that a point $p$ is a superattracting periodic point for a holomorphic map $f$ on $\mathbf{P}^{2}$ if there exists an $n \in \mathbf{N}$ such that $f^{n}(p)=p$ and both of the eigenvalues of the differential $d f^{n}(p)$ are equal to zero; i.e. $d f^{n}(p)$ is nilpotent.

We now prove Theorem 1.1.

Proof of Theorem 1.1, If $f$ is not 1 -critically finite, we are done by [2, Theorem 7.8]. Therefore, we can assume that $f$ is 1 -critically finite. Then by [6, Theorem 5.8], the Fatou limit set for $f$ consists of finitely many periodic critical points in $F_{2}$. It is obvious from the definition of $F_{2}$ that these periodic points belong to the singular set of $V=\bigcup_{j=0}^{\infty} f^{j}\left(C_{1}\right)$. Hence, arguing as in the first part of the proof of [2, Theorem 7.7], we are done. Note that the assumption of $\mathbf{P}^{2} \backslash V$ being hyperbolic in [2, Theorem 7.7] is only needed in the second part of its proof.

Remark 2.12. Bonifant and Dabija ([1, Theorem 4.1]) showed that an invariant critical component for a holomorphic map on $\mathbf{P}^{2}$ must be a rational curve. While most known examples of critically finite maps on $\mathbf{P}^{2}$ have only smooth rational curves as invariant critical components, here we give a family of critically finite maps on $\mathbf{P}^{2}$ with singular rational curves as invariant critical components:

$$
g_{d}:[z: w: t] \mapsto\left[z^{d}-w^{d-1} t:-w^{d}:-t^{d}\right], \quad d>2 .
$$

Note that $g_{d}$ maps the critical component $\{z=0\}$ to the singular rational curve $\left\{z^{d}=w^{d-1} t\right\}$ and maps $\left\{z^{d}=w^{d-1} t\right\}$ back to $\{z=0\}$. So $g_{d}^{2}$ will have $\left\{z^{d}=w^{d-1} t\right\}$ as a fixed critical component and obviously $g_{d}^{2}$ is a critically finite map.

\section{REFERENCES}

[1] Bonifant, A., Dabija, M.; Self-maps of $\mathbf{P}^{2}$ with invariant elliptic curves, Contemp. Math., vol. 311, Amer. Math. Soc., Providence, RI (2002), 1-25. MR1940161 (2004a:32029)

[2] Fornæss, J.E., Sibony, S.; Complex dynamics in higher dimension. I, Astérisque, 222 (1994), 201-231. MR 1285389(95i:32036) 
[3] Jonsson, M.; Some properties of 2-critically finite maps of $\mathbf{P}^{2}$, Ergodic Theory Dynam. Systems, 18 (1998), 171-187. MR1609475 (99b:32042)

[4] Milnor, J.; Dynamics in one complex variable, Princeton Univ. Press, 3rd. ed., 2006. MR2193309 (2006g:37070)

[5] Thurston, W.; On the combinatorics and dynamics of rational maps, preprint.

[6] Ueda, T.; Critical orbits of holomorphic maps on projective spaces, J. Geom. Anal., 8-2 (1998), 319-334. MR:1705160 (2000f:32026)

Department of Mathematics, Syracuse University, Syracuse, New York 13244

E-mail address: frong@syr.edu 